\title{
Reduction of the nonlinear phase shift induced by stimulated Brillouin scattering for bi-directional pumping configuration system using particle swarm optimization algorithm
}

\author{
H. A. Al-Asadi \\ hamid_alasadi@ieee.org
}

Computer Science Department, Faculty of Education of Pure Science, Basrah University, Basra, Iraq

We present a theoretical analysis of an additional nonlinear phase shift of backward Stokes wave based on stimulated Brillouin scattering in the system with a bi-directional pumping scheme. We optimize three parameters of the system: the numerical aperture, the optical loss and the pumping wavelength to minimize an additional nonlinear phase shift of backward Stokes waves due to stimulated Brillouin scattering. The optimization is performed with various Brillouin pump powers and the optical reflectivity values are based on the modern, global evolutionary computation algorithm, particle swarm optimization. It is shown that the additional nonlinear phase shift of backward Stokes wave varies with different optical fiber lengths, and can be minimized to less than 0.07 rad according to the particle swarm optimization algorithm for $5 \mathrm{~km}$. The bi-directional pumping configuration system is shown to be efficient when it is possible to transmit the power output to advanced when frequency detuning is negative and delayed when it is positive, with the optimum values of the three parameters to achieve the reduction of an additional nonlinear phase shift.

[DOI: http://dx.doi.org/10.2971/jeos.2013.13012]

Keywords: Nonlinear phase shift, stimulated Brillouin scattering, bi-directional pumping system, particle swarm optimization

\section{INTRODUCTION}

Stimulated Brillouin scattering (SBS) is an inelastic process, and can be described classically as acoustic waves travelling within the fiber core, causing moving periodic variations in the medium density that results in periodic variations in the refractive index and changes in the optical susceptibility through electrostriction. The optical properties of the optical fiber are characterized by linear and nonlinear susceptibilities. The lowest order nonlinearities are due to the third-order susceptibility. The pump wave scatters from this grating by Bragg diffraction and undergoes a frequency shift Stokes scattered wave due to the Doppler Effect [1].

Scattering occurs due to inhomogeneities of the refractive index or acoustic waves in the optical fiber. Brillouin scattering occurs due to inhomogeneities of the refractive index caused by phonons. The fluctuation of the Stokes wave at the higher Brillouin pump power can be attributed to the additional nonlinear phase shift caused by the spontaneous scattering process that initiates SBS and the gain/loss spectrum [2]-[4].

Nonlinear systems, unlike linear systems, must be treated in their full complexity and this usually requires analytical or numerical solutions and optimizations of the nonlinear equations. Generic meta-heuristic optimization algorithms are Evolutionary algorithms (EA) [5]. Swarm intelligence (SI) algorithms are population-based evolutionary algorithms. Particle Swarm Optimization (PSO) belongs to the category of (SI) [6] for solving single or multi-objective optimization problems with real-valued or discrete parameters [7]. PSO is a very successful swarm intelligence, as is well known, computationally inexpensive and can be implemented easily, since its CPU speed and memory requirements are low. Moreover, it does not require gradient information of the objective function but only its values [8].

In this paper, we first describe the model of SBS and the effect in the bi-directional pumping configuration system is considered, where the objective is to minimize an additional nonlinear phase shift of backward Stokes wave due to it. The performance of the evolutionary algorithm, Particle Swarm Optimization (PSO), and the analytical study results are compared for many different variations. We show from our analysis that the reduction of an additional nonlinear phase shift of backward Stokes wave depends on the optimal numerical aperture of the optical fiber, as well as the optical loss and the pumping wavelength.

\section{THEORETICAL ANALYSIS}

The electric fields of the optical pump wave, $E_{P}$ at a frequency $\omega_{P}$ propagating in the opposite direction of the backward scattered Stokes wave, $E_{S}$ is seeded at a frequency $\omega_{S}$, and the density wave, $\Delta \rho$, is given by [9]

$$
\begin{aligned}
& E_{P}(z)=\epsilon_{P}(z) F^{\rho} e^{i\left(K_{P} z-\omega_{P} t\right)} \\
& E_{S}(z)=\epsilon_{S}(z) F^{\rho} e^{i\left(-K_{S} z-\omega_{S} t\right)} \\
& \Delta \rho(z)=A(z) e^{i\left(K_{a} z-\omega_{a} t\right)}
\end{aligned}
$$

The monochromatic counterpropagating waves (the optical pump and the backward scattered Stokes) move along the fiber with slowly varying amplitudes $\epsilon_{P, S}(z)$ and the propagation constants $K_{P, S}=(n / c) \omega_{P, S}$, where $n$ is the core refrac- 
tive index, and $c$ is the velocity of light. $F^{P}$ is the electromagnetic field distribution with an arbitrary normalization. These two optical waves stimulate through electrostriction an acoustical wave, where $A$ is the amplitude of the density wave, and propagation constant $K_{a}$ with frequency $\omega_{a}=\omega_{P}-\omega_{S}$.

The change in electric fields can be ascribed to the system of three first order differential coupled equations thus [10],

$$
\begin{aligned}
\left(\frac{\partial}{\partial z}+\frac{\alpha}{2}\right) \epsilon_{P}(z) & =-\frac{i K_{P}}{4 \epsilon} \frac{\partial \epsilon}{\partial \rho} \epsilon_{S}(z) A(z) e^{i \Delta \omega t} \\
\left(\frac{\partial}{\partial z}-\frac{\alpha}{2}\right) \epsilon_{S}^{*}(z) & =-\frac{i K_{S}}{4 \epsilon} \frac{\partial \epsilon}{\partial \rho} \epsilon_{P}^{*}(z) A(z) e^{-i \Delta \omega t} \\
\left(\frac{\partial}{\partial z}+\frac{\Gamma_{B}}{v_{a}}\right) A(z) & =-\frac{i K_{a}}{4 \pi v_{a}^{2}} \rho_{0} \frac{\partial \epsilon}{\partial \rho} \epsilon_{P}(z) \epsilon_{P}(z) \epsilon_{S}^{*}(z) e^{-i \Delta \omega t}
\end{aligned}
$$

where, $\alpha$ is the optical loss coefficient in the fiber, $\frac{\partial \epsilon}{\partial \rho}$ is the change in permittivity $\epsilon$ with material density $\rho$, a frequency detuning $(\Delta \omega)$ between the counterpropagating optical waves exactly equal to $\Delta \omega=\omega_{S}+\omega_{B}-\omega_{P}$, where $\omega_{B}$ is the Brillouin frequency shift. $\epsilon^{*}$ denotes a complex conjugate. The acoustic wave is described by the acoustic velocity $v_{a}$ and the acoustic damping coefficient $\Gamma_{B}=\pi \Delta v_{B}$ (inverse of the acoustic phonon lifetime, $\tau_{B}$ ), where $\Delta v_{B}$, is the spectral width (FWHM) and is related to the acoustic phonon lifetime.

Eqs. (2a) and (2c) are further simplified by assuming that the acoustic phonon is heavily damped, and this allowed $A(z)$ to be eliminated, resulting in the following solutions:

$$
\begin{aligned}
\frac{\partial \epsilon_{P}(z)}{\partial z}=[ & -\frac{1}{8 \pi} \frac{i \omega_{P}^{2} K_{a} \rho_{0}}{v_{a} c^{2} K_{P}}\left(\frac{\partial \epsilon}{\partial \rho}\right)^{2} \\
& \left.\times\left(\frac{1}{-\Delta K v_{a}+i \pi \Delta v_{B}}\right)\left|\epsilon_{S}(z)\right|^{2}-\frac{\alpha}{2}\right] \epsilon_{P}(z) \\
\frac{\partial \epsilon_{S}^{*}(z)}{\partial z}=[ & -\frac{1}{8 \pi} \frac{i \omega_{S}^{2} K_{a} \rho_{0}}{v_{a} c^{2} K_{S}}\left(\frac{\partial \epsilon}{\partial \rho}\right)^{2} \\
& \left.\times\left(\frac{1}{-\Delta K v_{a}+i \pi \Delta v_{B}}\right)\left|\epsilon_{P}(z)\right|^{2}+\frac{\alpha}{2}\right] \epsilon_{S}^{*}(z)
\end{aligned}
$$

where $\Delta K=K_{P}+K_{S}-K_{a}$.

These equations reduce to the simpler system of coupled equations in terms of the optical intensities of the fields through the transformation $I_{P, S}=\left|\epsilon_{P, S}(z)\right|^{2} / A_{e f f}$, where $A_{\text {eff }}$ denotes the effective area defined as [11]:

$$
A_{e f f}=\frac{2 \pi\left[\int_{0}^{\infty}\left|F^{P}\right|^{2} \rho \partial \rho\right]^{2}}{\int_{0}^{\infty}\left|F^{P}\right|^{4} \rho \partial \rho}
$$

Solving Eq. (3b), in the case of negligible pump depletion, gives:

$$
\left|\epsilon_{S}(z)\right|^{2}=\left|\epsilon_{S}(L)\right|^{2} e^{\left(G_{B}-\alpha\right)(L-z)}
$$

where $\epsilon_{S}(L)$ is the Stokes field amplitude at the far end of optical fiber $(z=L)$ and the Brillouin gain $G_{B}$ is given by [12]

$$
G_{B}=\frac{1}{4 \pi} \frac{\omega_{S}^{2} K_{a} \rho_{0}}{v_{a} c 2}\left(\frac{\partial \epsilon}{\partial \rho}\right)^{2}\left[\frac{1}{\Delta K v_{a}-i \pi \Delta v_{B}}\right]\left|\frac{\epsilon_{P}^{2}}{K_{S}}\right|
$$

The Brillouin gain expression in Eq. (6) can be transformed to the constitutive parameters of the medium through the following relation:

$$
G_{B}=g_{B}\left[\frac{P_{P}}{A_{e f f}}\right]=\frac{2 \pi n 7 \rho_{12}^{2} K}{c \lambda_{P}^{2} v_{a} \rho_{0} \Delta v_{B}}\left[\frac{P_{P}}{A_{e f f}}\right]
$$

where $g_{B}$ is the peak Brillouin gain coefficient and $P_{P}$ is the optical pump power.

The effective area $A_{e f f}$ is dependent on the fiber geometry. By assuming a cylindrical single mode optical fiber, the model area is defined as $A_{e f f}=\pi r_{0}^{2}$, where $r_{0}$ (mode radius) is the 1 /e point of the amplitude field distribution. Mode radius can be deduced for a given fiber by calculating the fiber core radius $a$, and is [13]:

$$
r_{0}=a\left[0.632+\frac{0.522 \lambda_{P}^{1.5}}{(\pi a N A)^{1.5}}+\frac{0.0743 \lambda_{P}^{6}}{(\pi a N A)^{6}}\right]
$$

The sine of the maximum half-angle accepted by the optical fiber is called the numerical aperture $(N A)$ which is also governed by the core and cladding refractive indices.

The change in power due to SBS is dependent on the forward pump wave and the backward propagating Stokes wave as well as the additional nonlinear phase shift associated with the counterpropagating waves interaction. The electrical fields $E_{P, S}(z)$ and the additional nonlinear phase shifts $\Phi_{P, S}(z)$ can be expressed in terms of their powers $P_{P, S}(z)$ :

$$
E_{P, S}(z)=\sqrt{\frac{2 P_{P, S}(z)}{n \epsilon_{0} c A_{e f f}}} \exp \left(\mp \Phi_{P, S}(z)\right)
$$

Taking the imaginary parts from Eq. (9) to produce the additional nonlinear phase shift induced by SBS:

$$
\begin{aligned}
& \frac{\partial \Phi_{P}(z)}{\partial z}=\frac{1}{2} \phi \frac{g_{B}(v) P_{S}(z)}{A_{e f f}} \\
& \frac{\partial \Phi_{S}(z)}{\partial z}=\frac{1}{2} \phi \frac{g_{B}(v) P_{P}(z)}{A_{e f f}}
\end{aligned}
$$

where $\phi$ is given by

$$
\phi=\frac{2 \Delta v / \Delta v_{B}}{1+\left(2 \Delta v / \Delta v_{B}\right)^{2}} .
$$

\section{AN SBS-INDUCED ADDITIONAL NONLINEAR PHASE SHIFT USING THE SYSTEM WITH BI-DIRECTIONAL PUMP SCHEME}

The experimental set-up used to demonstrate an SBS-induced additional nonlinear phase shift using a bidirectional pumping configuration system (with an optical mirror at the far end of the optical fiber) is shown in Figure 1 [14]. It consists of: Tunable laser source (TLS), erbium doped fiber amplifier (EDFA), optical circulator (Cir), Single Mode Fiber Under Test (SMFUT), optical mirror and optical spectrum analyzer (OSA).

New analytical expressions for an additional nonlinear phase shift due to SBS with the bidirectional pumping configuration system were obtained [15]. The additional nonlinear 


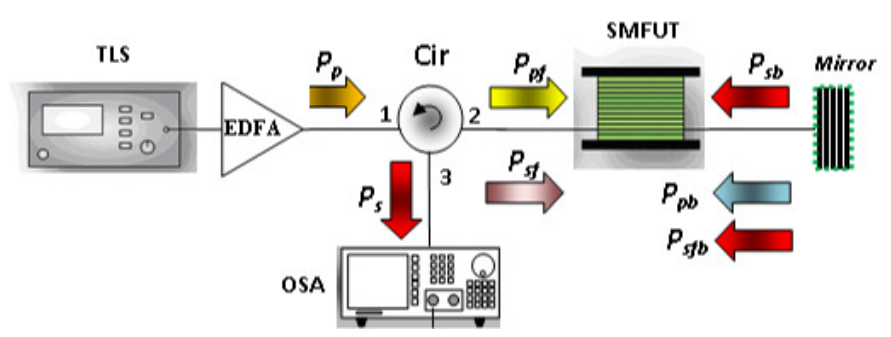

FIC. 1 The system with Bi-directional pumping scheme based on SMF fiber under test set to evaluation of stimulated Brillouin scattering effect.

phase shift for the Brillouin pump and the backward scattered Stokes powers assume the analytical formulations as:

$$
\begin{aligned}
& \Phi_{P}(z)= \\
& \phi\left[\begin{array}{l}
\frac{\alpha_{c b} R P_{s f}(0)}{\alpha} \exp \left(-\frac{g_{B} P_{p f}(0)\left(\exp \left(-\alpha \alpha_{c f} L\right)\right.}{\alpha A_{e f f}}\right. \\
\left.-\frac{\exp \left(-\alpha L\left(\alpha_{c f}+1\right)\right)}{\alpha A_{e f f}}-2 \alpha L\right)(\exp (\alpha z)-1) \\
-\frac{\alpha_{c f} \alpha_{c b} A_{e f f} P_{s b}(L)}{g_{B} P_{p f}(0)} \\
\exp \left(-\frac{g_{B} P_{p f}(0) \exp \left(-\alpha \alpha_{c f} L\right)}{\alpha \alpha_{c f} A_{e f f}}-\alpha L\right) \\
\left(\exp \left(\frac{g_{B} P_{p f}(0)}{\alpha \alpha_{c f} A_{e f f}}\right)-\exp \left(\frac{g_{B} P_{p f}(0) e^{\alpha z}}{\alpha \alpha_{c f} A_{e f f}}-\alpha L\right)\right)
\end{array}\right]
\end{aligned}
$$

$$
\begin{aligned}
& \Phi_{S}(z)= \\
& \phi\left[-\frac{1}{2} \frac{g_{B} P_{p f}(0)}{\alpha \alpha_{c f} A_{e f f}}\left(\exp \left(-\alpha \alpha_{c f} z\right)-\exp \left(-\alpha \alpha_{c f} L\right)\right)\right]
\end{aligned}
$$

where $P_{p f}(z), P_{p b}(z)$ represent the Brillouin pump power coupled into the fiber front end and the recycled pump power coupled into the other fiber end, respectively. $P_{s b}(z), P_{s f}(z)$, $P_{s f b}(z)$ are the backward Stokes wave power, the forward Stokes wave and the additional Stokes wave propagating again to the near end of the optical fiber as a function of fiber length, respectively. $\alpha_{c f}, \alpha_{c b}$ represent the loss factor of the circulator between ports 1 and 2 and between ports 2 and 3 , respectively. The reflectivity of the optical mirror is $R$.

\section{PARTICLE SWARM OPTIMIZATION}

For highly nonlinear and combinatorial optimization problems, the conventional methods are facing difficulties of finding global or close to global optimum points in ultra-large search spaces. Evolutionary algorithms are a popular class of stochastic optimization methods to locate the global optimal solution. Particle Swarm Optimization (PSO) is a populationbased global optimization scheme that belongs to the category of Swarm Intelligence for solving many hard optimization problems in both static and dynamic environments. PSO, first introduced by Kennedy and Eberhart, is one of the modern evolutionary computation algorithms [16].

There are four vectors: the position $X_{i}$; their previous best position $P_{i}$, the best position explored so far is $g_{i}$ and the velocity $V_{i}$, with a dimensionality equal to one of the problem spaces for each individual $i$ in a particle swarm. An objective (fitness) function is used to quantisize the quality of the solution represented by each particle in a specific manner as follows. The particle having the index of the best particle among all the particles is treated as global best particle, and is marked as the global-best particle, i.e, gbest $_{i}$.

To generalize, the original process for implementing PSO is given as:

- Randomly initialize particle positions and velocities.

- While not terminating.

- For each particle $i$

- Evaluate objective function, $Y_{i}$ at current position $X_{i}$.

- If $Y_{i}$ is better than $i$-th local best then update $i$-th local best and $P_{i}$.

- If $Y_{i}$ is better than gbest $_{i}$ then update gbest $_{i}$ and $g_{i}$.

- Update velocity $V_{i}$ and position $X_{i}$ using,

$$
\begin{aligned}
& v_{i j}^{k}=w v_{i j}^{k-1}=c_{1} r_{1}\left(p b_{i j}^{k-1}-x_{i j}^{k-1}\right)+c_{2} r_{2}\left(g b_{j}^{k-1}+x_{i j}^{k-1}\right) \\
& x_{i j}^{k}=x_{i j}^{k-1}+v_{i j}^{k}
\end{aligned}
$$

- Repeat until optimal solution or solution of desired quality is found.

The main variants in PSO are: $w$ is the intertia weight factor, $c_{1}$ and $c_{2}$ represent the weighting of the stochastic acceleration constants, and $r_{1}$ and $r_{2}$ are two random functions.

Depending on Eq. (11), the additional nonlinear phase shift due to SBS characteristics is known in the bi-directional pump configuration system. The objective function is defined as the minimization function using Eqs. (4), (7), (8) and (11). Our aim in this paper is to find optimum parameters usually chosen to reduce the additional nonlinear phase shift backward Stokes wave through SBS, sine of the maximum half-angle accepted by the optical fiber $(N A)$, pumping wavelength $\left(\lambda_{p}\right)$ and optical loss coefficient $(\alpha)$. Values of parameters in this investigation, involved in the optimization process, are chosen randomly as shown in Table 1.

\begin{tabular}{|c|c|c|}
\hline Parameter & Unit & Value \\
\hline$N A$ & & $0.05-2$ \\
$\lambda_{p}$ & $(\mathrm{~nm})$ & $1545-1558$ \\
$\alpha$ & $(\mathrm{dB} / \mathrm{km})$ & $0.19-0.25$ \\
\hline
\end{tabular}

TABLE 1 Ranges of parameter values used in optimization algorithm.

\section{RESULTS AND DISCUSSIONS}

The Parameter values needed to run the optimization program (implemented in MATLAB) are given in Table 2. For a TLS linewidth $\Delta v=10 \mathrm{MHz}$ and $\lambda_{p}=1550 \mathrm{~nm}$, the equivalent injected Brillouin pump power, therefore, turns out to be in the range $0-30 \mathrm{~mW}$. The output from the laser running single frequency was launched, via an EDFA and optical circulator, into the SMF used and are 3, 5, 7, 9, 11, 13, 15 and $17 \mathrm{~km}$ of single core fiber for the reflectivity of optical mirror $R=0.75$. Typical values for the experimental Bi-directional pumping configuration system shown schematically in Figure 1, used 


\begin{tabular}{|c|c|}
\hline Parameter & Value \\
\hline Particle swarm size & 70 \\
Iteration number range & $100-1000$ \\
Acceleration constant & 2 \\
Initial velocity & 0 \\
Initial local and global best & 0 \\
Initial inertia weight & 0.5 \\
\hline
\end{tabular}

TABLE 2 Required information of PSO.

to calculate the additional nonlinear phase shift due to SBS effect, are: $\alpha=4.5 \mu \mathrm{m}, g_{N}=2 \cdot 10^{-11} \mathrm{~m} / \mathrm{W}, \alpha_{c 1}=0.8 \mathrm{~dB}$, $\alpha_{c} 2=0.9 \mathrm{~dB}, v_{B}=11 \mathrm{GHz}, \Delta v_{B}=30 \mathrm{MHz}, P_{s f}(0)=1 \mathrm{nW}$, $P_{s b}(0)=0.5 \mathrm{nW}$.

The most important feature of the configuration under study is examined: the additional nonlinear phase shift induced by SBS. The optimum additional nonlinear phase shift of the backward scattered Stokes wave depends on the optimal values of numerical aperture and pumping wavelength parameters (using particle swarms algorithm) with different lengths of optical fiber, as shown in Figure 2. Calculated values of optimal optical loss with different optical fiber lengths are given in Table 3. To arrive at an analytical solution, initial values are re-

\begin{tabular}{|c|c|}
\hline $\begin{array}{c}\mathrm{L} \\
(\mathrm{km})\end{array}$ & $\begin{array}{c}\alpha \\
\mathrm{dB} / \mathrm{km}\end{array}$ \\
\hline 3 & 0.1923 \\
\hline 5 & 0.1941 \\
\hline 7 & 0.1944 \\
\hline 9 & 0.1948 \\
\hline 11 & 0.1952 \\
\hline 13 & 0.1952 \\
\hline 15 & 0.1954 \\
\hline 17 & 0.1955 \\
\hline
\end{tabular}

TABLE 3 Calculated $\alpha_{\text {optimal }}$ for different optical fiber lengths.

quired for each of the additional nonlinear phase shifts of two optical counterpropagating waves. The additional nonlinear phase shift for both the pump and Stokes waves are known, but one condition is known at $z=0$ and the other at $z=L$, as $\Phi_{P}(0)=0, \Phi_{S}(L)=0$. From Figure 2, it can be seen that, as the numerical aperture is increased, the optimum additional nonlinear phase shift of the backward scattered Stokes wave decreases. $\Phi_{S}(z)$ values also start to increase in accordance with the increased optimum pumping wavelength. The values of $\Phi_{S}(z)$ start to decrease from its maximum value of $-0.065 \mathrm{rad}$, $-0.128 \mathrm{rad}$, and $-0.192 \mathrm{rad}$ and so forth until it reaches the lowest value at $N A=1.488$ with different Brillouin pump powers, 10,20 and $30 \mathrm{~mW}$, respectively.

To test the range of the additional nonlinear phase shift of backward Stokes wave over which the Brillouin pump power increases, the results from the analytical solution and objective function will be compared to the results of the analytical solution without using optimal parameter values in Figure 3. The additional nonlinear phase shift is seen to decrease linearly as the pump power is increased, in both cases with different optical fiber lengths, 5, 11 and $17 \mathrm{~km}$. Based on the
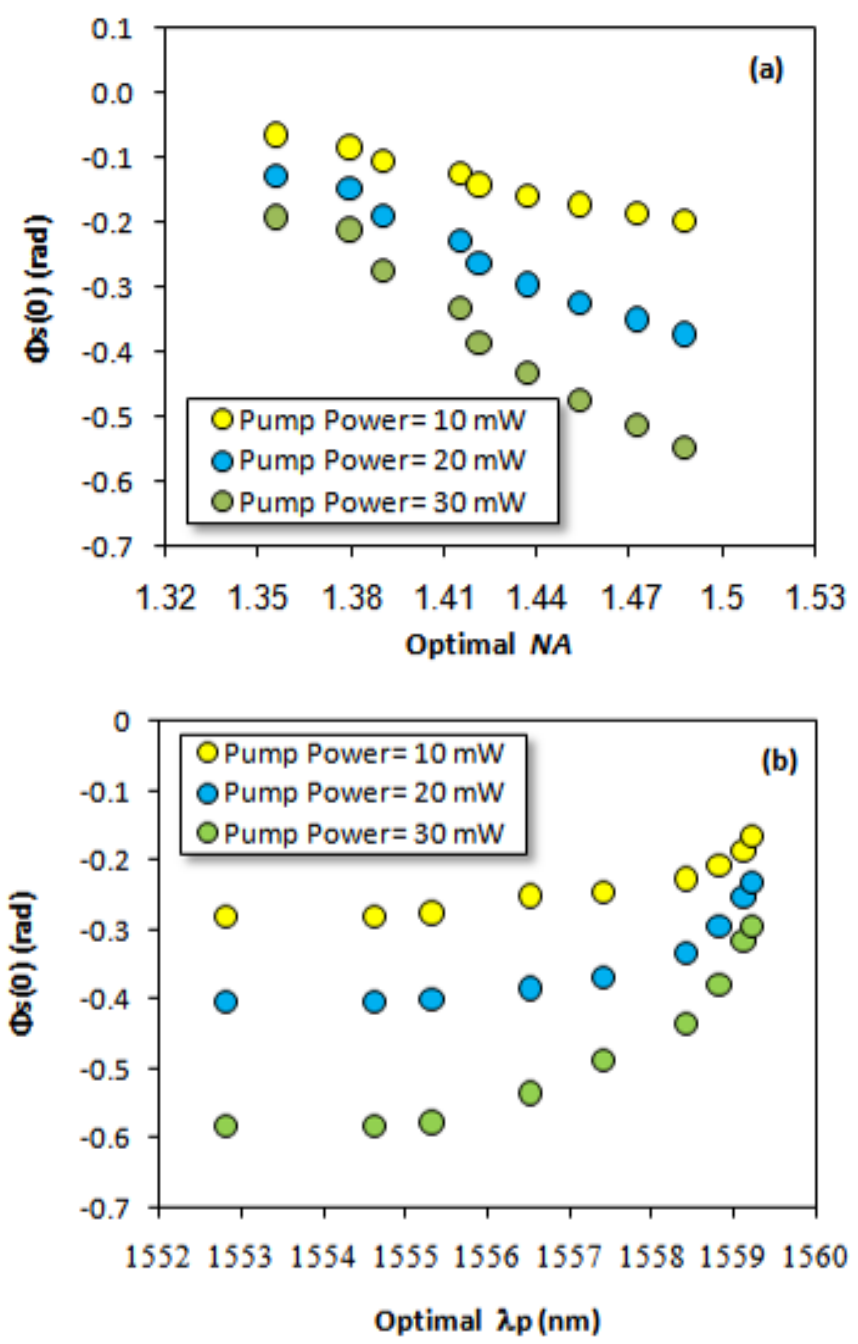

FIG. 2 Dependencies of the additional nonlinear phase shift of backward Stokes waves in SMF using Bi-directional configuration system on (a) optimal numerical aperture, and (b) optimal pumping wavelength.

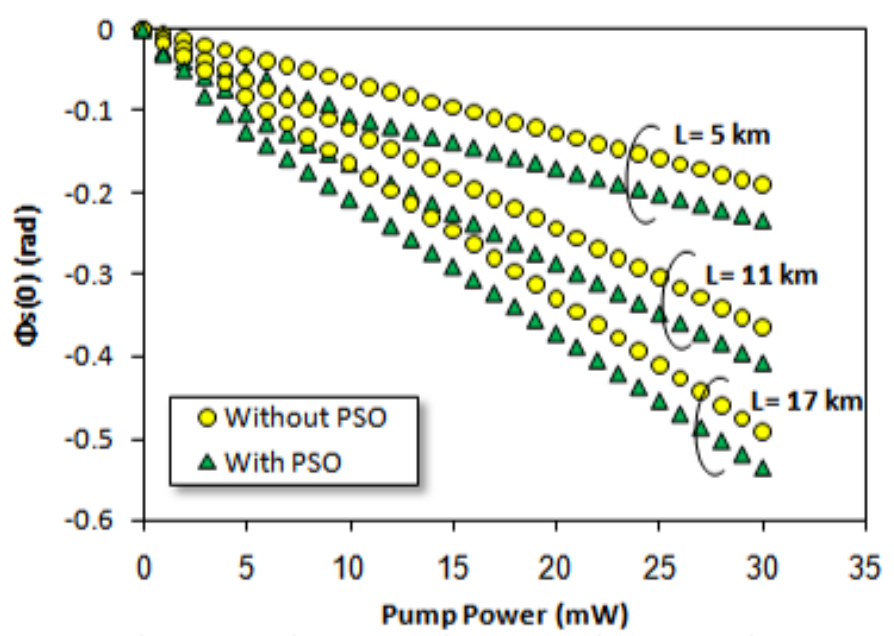

FIG. 3 Additional nonlinear phase shift of backward Stokes waves plotted as a function of the Brillouin pump power for three different optical fiber lengths. The circle mark and the triangle mark represent the analytical results using PSO and without using it, respectively.

findings, Figure 3 shows clearly that at low Brillouin pump 

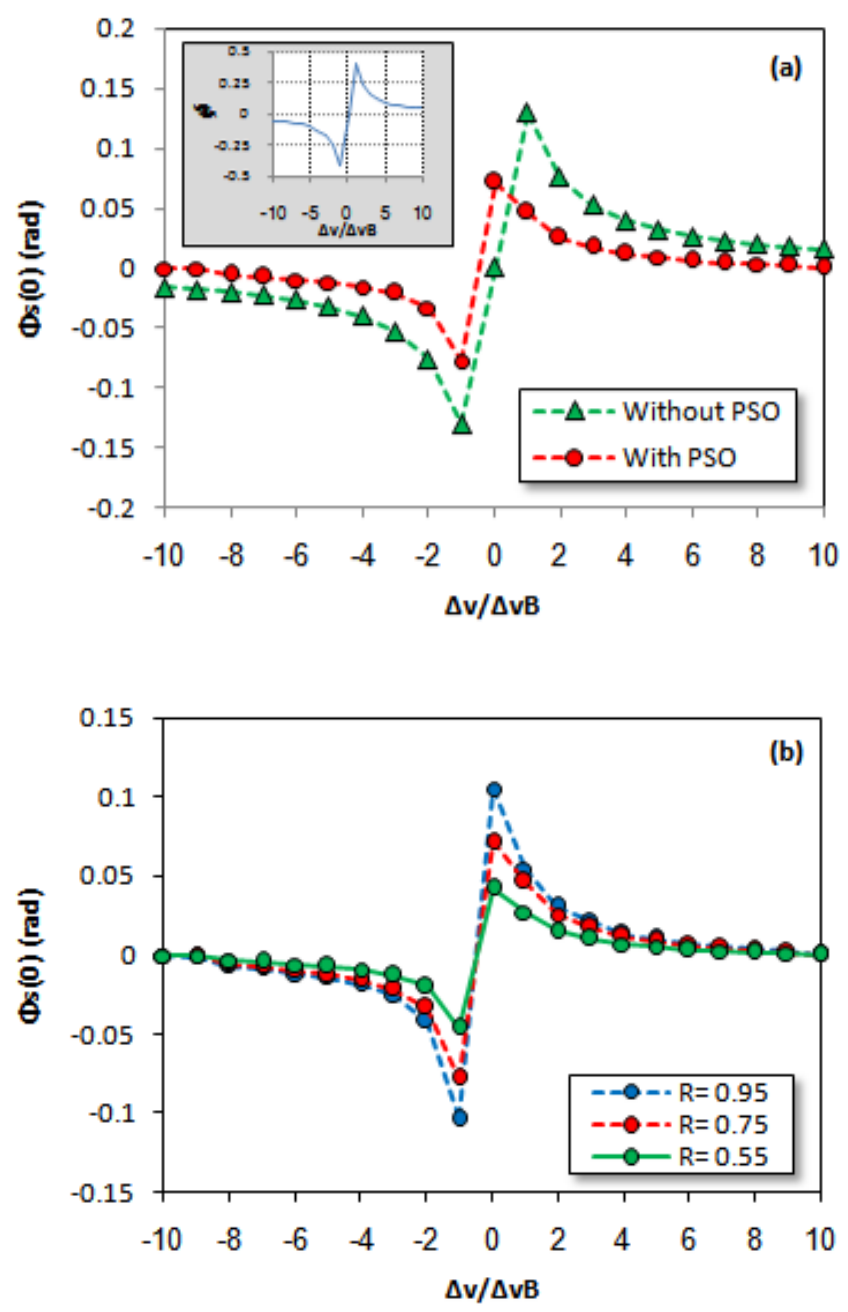

FIG. 4 Results of the optimal additional nonlinear phase shift of backward Stokes waves compared to the conventional results as a function or relative frequency detuning at $L=5 \mathrm{~km} . R=0.75$, (b) optimal additional nonlinear phase shift of backward Stokes waves as a function or relative frequency detuning for different optical reflectivity values at $L=5 \mathrm{~km}$.

power, the additional nonlinear phase shift decreases slowly until the threshold is reached. Beyond the threshold, the increment of Brillouin pump power increases the backward Stokes on the Bi-directional pumping configuration system through the additional gain provided by the recycled forward Brillouin pump power by the optical mirror at the far end of the optical fiber to the near end of the optical fiber where the reflectivity is 0.75. Due to a reduction in the Brillouin threshold power, the saturation level for the output pump power is also reduced, and has the same effect as increasing the Brillouin gain and decreasing the Brillouin loss.

For low pump powers, the additional nonlinear phase shift of backward Stokes power using optimal values of numerical aperture, laser wavelength and optical loss and without using the PSO algorithm, predict that both simulation results are close to each other, with an error ratio of less than $4 \%$, as shown in Figure 3. As the Brillouin pump power is increased more than $8 \mathrm{~mW}$, the error ratio between these two values is less than $10 \%$. Here, it can be seen that the optimum point for minimum additional nonlinear phase shift of backward
Stokes power can be obtained if the configuration under study operates with a numerical aperture $N A=1.488$ and pumping wavelength $\lambda_{P}=1555.3 \mathrm{~nm}$, and optimal optical fiber loss is equal to 0.192 at $-0.233,-0.407$ and -0.536 rad at 5,11 and $17 \mathrm{~km}$ optical fiber length, respectively.

The bi-directional flow of Brillouin pump power is important in this configuration system because bi-directional flow of power will reduce the system threshold power to half as compared to a uni-directional flow. For a system with a bidirectional pumping configuration as used in this paper, a reduction in the additional nonlinear phase shift of backward Stokes wave level is expected (hence higher additional gain and low threshold power). This is again illustrated using the configuration with a bi-directional flow which compared the additional nonlinear phase shift with and without the PSO algorithm dependence on the detuning frequency, as shown in Figure 4 .

The results are calculated for Brillouin pump power, optical fiber length and optical reflectivity of $30 \mathrm{~mW}, 5 \mathrm{~km}$ and 0.75 . Comparing the two graphs presented in Figure 4(a), it can be seen that the additional nonlinear phase shift of backward Stokes wave level is the lowest of all when the PSO algorithm is used as compared to results without using it. At high pump power, the Stokes wave grows very quickly near the front of the fiber, resulting in a short effective length. A significant reduction in additional nonlinear phase shift of backward Stokes wave can be gained by using a different reflectivity mirror, as shown in Figure 4(b). This illustrates the efficiency of the bi-directional pumping configuration system in which it is possible to transmit the power output to advanced when $\Delta v$ is negative $\left(v>v_{S}+v_{B}\right)$ and delayed when $\Delta_{v}$ is positive $\left(v<v_{S}+v_{B}\right)$, and the additional nonlinear phase shift of backward Stokes wave due to SBS calculated analytically to less than 0.07 rad using the PSO algorithm.

\section{CONCLUSION}

We have studied theoretically the stimulated Brillouin scattering in a bi-directional configuration system, and have highlighted the additional nonlinear phase shift of backward Stokes waves induced through it. For a single mode optical fiber we have shown that the additional nonlinear phase shift of backward Stokes waves is explicitly dependent on the fiber length, numerical aperture and the pumping wavelength. Our results show that the minimum additional nonlinear phase shift of backward Stokes waves is less than $0.07 \mathrm{rad}$ at optimal values $N A=1.48, \lambda_{P}=1555.3 \mathrm{~nm}$, and $\alpha=0.19 \mathrm{~dB} / \mathrm{km}$, utilizing an efficient and reliable method called the particle swarm optimization algorithm at optical fiber length equal to $5 \mathrm{~km}$.

\section{References}

[1] G. P. Agrawal, Nonlinear Fibre Optics (fourth edition, Academic Press, New York, 2006). 
[2] R. W. Boyd, Nonlinear Optics (second edition, Academic Press, New York, 2002).

[3] D. Cotter, "Stimulated Brillouin scattering in optical fibers," J. Opt. Commun. 4, 10-19 (1982).

[4] A. Yeniay, J. M. Delavaux, and J. Toulouse, "Spontaneous and stimulated Brillouin scattering gain spectra in optical fibers," J. Lightwave Technol. 20, 1425-1432 (2002).

[5] E. Elbeltagi, T. Hegazy, and D. Grierson, "Comparison among five evolutionary-based optimization algorithms," Adv. Eng. Inform. 19, 43-53 (2005).

[6] C. Blum, and D. Merkle (Eds.), Swarm Intelligence: Introduction and Applications (Springer, Berlin / Heidelberg, 2008).

[7] C. Susana, and A. Coello, Particle Swarm Optimization in Nonstationary Environments (Springer, Berlin / Heidelberg, 2009).

[8] E. Bonabeau, M. Dorigo, G. Theraulaz, Swarm Intelligence: From Natural to Artificial Systems (Oxford University Press, Oxford, 1999).

[9] R. W. Boyd, K. Rzazewski, and P. Narum, "Noise initiation of stimulated Brillouin scattering," Phys. Rev. A 42, 5514-5520 (1990).

[10] R. B. Jenkins, R. M. Sova, and R. I. Joseph, "Steady-state noise analysis of spontaneous and stimulated Brillouin scattering in optical fibers," J. Lightwave Technol. 25, 763-770 (2007).

[11] M. Artiglia, "Mode field diameter measurements in single-mode optical fibers," J. Lightwave Technol. 7, 1139-1152 (1989).

[12] M. Nikles, L. Thevenaz, and P. A. Robert, "Brillouin Gain spectrum characterization in single-mode optical fibers," J. Lightwave Technol. 15, 1842-1851 (1997).

[13] Y. Namihira, "Relationship between nonlinear effective area and modefield diameter for dispersion shifted fibers," Electron. Lett. 30, 262-263 (1994).

[14] M. Ajiya, M. A. Mahdi, M. H. Al-Mansoori, Y. G. Shee, S. Hitam, and M. Mokhtar, "Reduction of stimulated Brillouin scattering threshold through pump recycling technique," Laser Phys. Lett. 6, 535538 (2009).

[15] H. A. Al-Asadi, A. A. Bakar, F. R. Adikan, and M. A. Mahdi, "Analytical study of nonlinear phase shift through stimulated Brillouin scattering in single mode fiber with the pump power recycling technique," J. Opt. 13, 105701 (2011).

[16] J. Kennedy, R. C. Eberhart, and Y. Shi, Swarm Intelligence (Morgan Kaufmann Publishers, San Francisco, 2004). 